\title{
EFFECTS OF REAL AND SIMULATED WEIGHTLESSNESS ON THE CARDIAC AND PERIPHERAL VASCULAR FUNCTIONS OF HUMANS: A REVIEW
}

\author{
HUI ZHU ${ }^{1}$, HANQING WANG ${ }^{2}$, and ZHIQIANG LIU ${ }^{1}$ \\ ${ }^{1}$ Central South University, Changsha, Hunan, China \\ School of Energy Science and Engineering \\ ${ }^{2}$ Hunan University of Technology, Zhuzhou, Hunan, China \\ Collaborative Innovation Center for Energy-conservation in Buildings and Environment Control
}

\begin{abstract}
Weightlessness is an extreme environment that can cause a series of adaptive changes in the human body. Findings from real and simulated weightlessness indicate altered cardiovascular functions, such as reduction in left ventricular (LV) mass, cardiac arrhythmia, reduced vascular tone and so on. These alterations induced by weightlessness are detrimental to the health, safety and working performance of the astronauts, therefore it is important to study the effects of weightlessness on the cardiovascular functions of humans. The cardiovascular functional alterations caused by weightlessness (including long-term spaceflight and simulated weightlessness) are briefly reviewed in terms of the cardiac and peripheral vascular functions. The alterations include: changes of shape and mass of the heart; cardiac function alterations; the cardiac arrhythmia; lower body vascular regulation and upper body vascular regulation. A series of conclusions are reported, some of which are analyzed, and a few potential directions are presented.
\end{abstract}

Key words:

Weightlessness, Cardiac functions, Peripheral vascular functions

\section{INTRODUCTION}

Cardiovascular system of humans has adapted to Earth's gravity of $1 \mathrm{G}$ over millions of years. When exposed to weightlessness, however, the cardiovascular functions change considerably in respect of blood redistribution, elevated compliance of the lower body veins, contractility of the heart, cardiac arrhythmia and orthostatic intolerance, all of which may be detrimental to the health of the astronauts. It has been reported that one of the astronauts on Apollo 15 suffered myocardial infarction after spaceflight, and another astronaut on Soyuz had to go back to Earth due to the severe cardiac arrhythmia.

As the rapid development of space exploration continues, manned spacecrafts are increasingly preferred. The United States and Russia have launched their manned spacecrafts successfully since last century. What's more,

The article was financially supported by National Natural Science Foundation of China (Grant No. 51276057; 51376198, grant manager: Prof. Hanqing Wang, Prof. Zhiqiang Liu) and Hunan Provincial Innovation Foundation for Postgraduate (Grant No. CX2014B065, grant manager: Hui Zhu, PhD).

Received: April 21, 2014. Accepted: January 24, 2015.

Corresponding author: H. Wang, Hunan University of Technology, School of Civil Engineering, Taishan West Road 88, Zhuzhou, Hunan Province, 412001, China (e-mail: hqwang2011@126.com). 
the spaceflight duration is increasing and the activities of the astronauts in spacecraft nowadays are much more frequent, especially the extra-vehicular activities (EVA). Therefore, more challenges will be faced by the cardiovascular system of the astronauts, and researches on the cardiovascular system in weightlessness are of great significance since it is important to ensure that the astronauts are in good working performance and remain healthy and safe during the spaceflight.

Since the early 1960s, studies on the effects of weightlessness on human cardiovascular system during spaceflights have produced a great number of conclusions. Meanwhile, terrestrial experiments with simulated weightlessness have also provided an amount of meaningful information. Based on the studies in the latest decade, the effects of weightlessness on human cardiovascular functions are reviewed, to explore and develop an understanding of the physiological challenges faced by the astronauts when working or living in weightlessness (prolonged weightlessness in particular).

\section{EFFECTS OF THE WEIGHTLESSNESS ON HEART}

\section{Shape and mass of the heart}

The shape of the heart will change in weightlessness, which is demonstrated by the cardiac sphericity and the Geometric Aspect Ratios (GAR, length to width quotient). According to the finite element simulation, the GAR of the left ventricle decreases by $3.65 \%$ in weightlessness compared to the value in terrestrial conditions, which results in the changes of the radius of curvature of the heart $[1,2]$. The conclusion has been verified by the data from spaceflight. During the spaceflight, diastolic dimensions of the left ventricle of 4 astronauts were measured with echocardiography first, then the dimensions were translated into circularity indices and GAR. The results indicate that the average circularity index increases by $4.1 \%$ while the GAR decreases by $5.3 \%$ [3]. These findings suggest that the heart is more spherical in weightlessness.
In addition to the shape, the mass of the heart also changes in weightlessness. Bed rest experiment was carried out by Perhonen et al. to evaluate the cardiac mass in simulated weightlessness [4]. In the experiment, 5 subjects were in horizontal bed rest for 6 weeks, and 3 of the 5 subjects continued the experiment till the end of the 12th week. And the ventricular mass was obtained by means of Magnetic Resonance Imaging (MRI) with Simpson's rule technique. The results indicate that the mass of the left and right ventricle decreases by $8 \pm 2.2 \%$ and $10 \pm 2.7 \%$ respectively, and the wall thickness of the left ventricle decreases by $4 \pm 2.5 \%$ [4] after 6 weeks of bed rest $(\mathrm{N}=5)$. In addition to the evidences from the bed rest experiment, the findings during the spaceflights also prove that the cardiac mass is decreasing. Early in 1977, Henry et al. [5] reported a mass reduction of the left ventricle in 3 astronauts during the Skylab-4 mission (from November 16, 1973 to February 8, 1974). Later, during the 2nd German Spacelab mission Spacelab D2 in 1993-1994 the average left ventricular mass decreased by $12 \pm 6.9 \%$ among the 4 astronauts. In recent years, the echocardiographic measurements of left ventricular mass in astronauts have been carried out, and the results demonstrate a comparative $9.1 \%$ reduction in post-flight left ventricular (LV) mass that returned to preflight values by the 3rd day of recovery [6]. The phenomenon is attributed to the loss of fluid volume, although according to some other opinions, the LV mass reduction in spaceflight is due to the loss of myocardial mass $[7,8]$.

\section{Cardiac function alterations}

It has been proved that the heart rate, cardiac output, systolic and diastolic pressure are influenced by weightlessness. During spaceflight, the heart rate is lower than that on Earth (standing posture). Heart rate was measured on International Space Station (ISS) with electrocardiogram (ECG) among 11 astronauts during a 6-month mission [9]. The consecutive RR-intervals recorded by 
the ECG were averaged to calculate the mean heart rate. The results show that mean heart rate in spaceflight equals the pre-flight supine values, but is lower compared with pre-flight standing posture (both: $\mathrm{p}<0.05$ ).

Interesting results were obtained in studies of a Russian astronaut Polyakov who had stayed on the MIR space station for 438 days [10]. In the experiment, heart rate was assessed by the beat-to-beat interval that was recorded by ECG. During the 1st month, a lengthening of beat-tobeat interval by $82 \mathrm{~ms}$ was observed, and a further 94-ms increase of the interval was observed after 6 months (with no further change afterwards). This increase in the average beat-to-beat interval suggests a reduction of heart rate by about $20 \%$.

Then how does the heart rate change with the duration of the spaceflight? Early in 1996 Charles pointed out that the mean heart rate of 26 astronauts during the long-term spaceflight (as long as 12 months) tended to increase as the duration of the spaceflight extended [11]. (The elevated heart rate may be a response to the hypovolemia in long-duration spaceflight). Although this opinion has been widely accepted, what deserves attention is that there were only 2-3 astronauts participating in the spaceflight after the 7 th month.

In addition, weightlessness has considerable effects on the cardiac output, systolic and diastolic pressure. It has been commonly accepted that the cardiac output increases slightly, while the systolic and diastolic pressure decrease in weightlessness. Echocardiographic data collected from ISS during a 114-day mission among 6 astronauts support this point of view [12]. Similarly, measurements conducted by Hughson in 2012 on the ISS during a 6-month mission $(\mathrm{N}=6)$ also point to the same conclusion [13]. Moreover, attenuation of the cardiac contractile function, which has also been noticed by many other researchers such as Carvil [14], Zuj [15] and Platts [16], has been observed in weightlessness, although the mechanism still needs further investigating.

\section{Cardiac arrhythmia}

The cardiac arrhythmia, such as the premature ventricular contractions (PVCs), premature atrial contractions (PACs) and prolongation of the time interval between the start of the $\mathrm{Q}$ wave and the end of the T wave in the heart's electrical cycle (QT interval prolongation), is often observed in spaceflights and head down tilt (HDT) experiments. In earlier days, PVCs and PACs were believed to be caused by the psychological factors of the astronauts, instead of the weightlessness per se [17]. For example, PVCs experienced by 9 astronauts in 42 spaceflights between 1964 and 1985 were ascribed to the psychological stresses [18]. Later on, physical stress and potassium deficiency in weightlessness were proved to be the causes of cardiac arrhythmia. During the Apollo 15 mission, astronaut James Benson Irwin suffered bigeminal PVCs and premature atrial contractions (PACs). The cause of those arrhythmias was found to be related to a decrease in the total body potassium level and the heavy workload on the surface of the Moon [19].

Recent findings suggest that the lack of gravitational stress during long-term spaceflights is associated with the adaptive changes in cardiovascular structure and neurohumoral control circuits. These changes increase the probability of cardiac arrhythmias and eliminate the ability of the cardiovascular system to cope with gravitational stress post-flight [20].

In addition to PVCs and PACs, QT interval prolongation is another common cardiac arrhythmia in real and simulated weightlessness. Generally speaking, QT interval changes little in short-duration spaceflight (5-10 days, $\mathrm{N}=11$, male), and the QT interval prolongation occurs between the 9th and 30th day of spaceflight [21]. This observation has been verified by Anzai [22]. The average QT interval was found to be longer than 0.45 in $24 \%$ of the astronauts on MIR Station and ISS, which suggested that the ventricular repolarization process might be altered during longduration spaceflight [23]. 
What's more, the QT interval prolongation may increase the risk of torsades de pointes (TdP) that can result in myocardial repolarization disorder [24]. According to the recent findings, there are 3 possible causes of the QT interval prolongation in weightlessness. First, higher incidence of bradycardia during long-duration spaceflights rather than short-duration spaceflights can result in QT interval prolongation. Second, changes in the autonomic nervous system (such as a higher tension of the vagus nerve) caused by weightlessness can also affect the QT interval $[25,26]$. Third, cardiac function alterations induced by weightlessness, such as the reduced contractile function, may also influence the QT interval prolongation [27]. And finally the medication usage during the space missions such as ciprofloxacin, haloperidol, propranolol and nortriptyline may also affect the QT interval.

Except PVCs, PACs and QT interval prolongation, there are other kinds of cardiac arrhythmias, such as short-duration atrial fibrillation and non-sustained ventricular tachycardia. It has been reported that ventricular tachycardia (VT) was recorded on MIR station with maximum rate of 215 beats per min during a long-duration spaceflight. Possible cause could be the change in the ventricular mass or volume, an electrolyte disturbance and autonomic alterations due to weightlessness [28].

A series of possible causes of the cardiac arrhythmias have been put forward in the latest decade, including the underlying heart diseases of the astronauts, unbalance of the electrolyte (such as lower potassium level in human body), increased catecholamine secretion, high tension of the vagus nerve. Evidences from the recent animal experiments have indicated that the myocardial connexin reconstruction occurs under simulated weightlessness, which results in the abnormality of the cardiac electrical conductance and formation of the microreentry. As a result, the cardiac arrhythmia occurs [29]. However, further study is needed to examine whether the same phenomenon happens in humans.

\section{EFFECTS OF WEIGHTLESSNESS ON PERIPHERAL VESSELS}

\section{Lower body vascular regulation}

Dynamic regulation of the lower body vascular system is one of the adaptive changes occurring in human body during weightlessness. However, the weightlessness has inconspicuous influences on the arterial system due to its wall thickness, and the regulation is mainly reflected by the alteration of the vascular resistance and the vascular tone.

Spaceflight extension can weaken the lower body arterial resistance and affect the regulation of the vascular tone. Further, the Lower Body Negative Pressure (LBNP) test has been conducted during different periods of the prolonged spaceflight to investigate the hemodynamic characteristics of the cerebral circulation. In the test, 15 subjects were put into the negative pressure device which pulls the blood of the subjects from the upper body to the lower body, meanwhile the hemodynamic parameters were measured with echography and Doppler ultrasonic flowmetry. A cerebral circulation deficiency was observed through the LBNP test. Besides, during the LBNP test, a decline of the gravity-dependent reactions was also observed, which was a function of the spaceflight duration [30]. In addition, measurements on the MIR station among 6 astronauts during a 6-month mission were conducted. The results indicate that lower-limb resistance decreases $(-5 \%$ to $-18 \%, \mathrm{p}<0.05)$ throughout the flights, and femoral vein cross-sectional area is enlarged after the 1st week of the spaceflight $(+15 \%$ to $+35 \%, \mathrm{p}<0.05)$ [31].

Another important change of the artery in weightlessness is the alteration of the arterial vascular tone. Reduction of the constrictive ability of large blood vessels of the lower extremities has been observed during spaceflight, which impairs the regulation of the artery and finally decreases the orthostatic stability [32]. It has been reported recently that the vascular tone of the lower-limb arteries of the astronauts is in decline after working and living in ISS 
for 6 months [33]. The alteration of the lower-limb artery tone is a function of the spaceflight extension, which is found to be reduced in short-term spaceflight while elevated in long-term spaceflight [7,34]. Alteration in arterial tone is believed to be caused by the local remodeling within vascular wall, and it may play a significant role in weightlessness-induced orthostatic intolerance [35].

The influence of the weightlessness on lower-limb veins is more profound, including the changes in blood filling, vascular compliance and the veins capacity. The blood filling is in decline and the blood flow in the lower extremities decreases, due to the weightlessness induced blood redistribution. Measurements with venous occlusion plethysmograph (VOP) on 7 astronauts preflight, in-flight and post-flight were conducted. The results showed that the calf blood flow decreased by $41 \%$ in weightlessness (to $1.15 \pm 0.16 \mathrm{ml} \times 100 \mathrm{ml}^{-1} \times \mathrm{min}^{-1}$ ) relative to terrestrial supine conditions $\left(1.94 \pm 0.19 \mathrm{ml} \times 100 \mathrm{ml}^{-1} \times \mathrm{min}^{-1}\right.$, $p=0.01$ ) [36]. However, there are different findings. During an 84-day mission on Skylab-4, the blood flow of 2 astronauts ( 3 in total) at rest was found to increase by $100-500 \%$, between day 13 to day 76 [37]. This could be the result of the body fluid compensation caused by hypovolemia in weightlessness.

In addition to the blood filling, compliance of the lowerlimb veins is also affected by weightlessness. It has been verified that the compliance of the lower-limb veins elevates at the beginning of spaceflight, then decreases with the spaceflight extension and keeps at a certain level that is higher than preflight values. Data from the spaceflight have proved the notion. During a 6-month spaceflight, increased leg veins dilation and compliance were persistently observed among 8 astronauts from the early period of spaceflight (weeks 1-2), and the increase was still considerable till months 2-3 of exposure to weightlessness and tended to stabilize at the end of the 6th month [38]. Changes in the compliance of the lower-limb veins in weightlessness are associated with the altered structure and function of the vessels, and to some extent due to the decreased muscle tone. However, evidences from other spaceflights and $-6^{\circ}$ HDT experiments lead to the opposite conclusions. For example, by blocking the vein of the calf, Watenpaugh [36] found that venous compliance of lower limbs increased only at the 1st week after spaceflight. And a 120-day $-6^{\circ}$ HDT experiment was carried out by Christ, during which 6 subjects were placed on a modified bed that sustained $-6^{\circ}$ HDT and this posture was continuously maintained throughout the 120 days. In the experiment, venous congestion strain gauge plethysmography (VCP) technique was employed to evaluate the hemodynamic characteristics. The results also suggest that after 118 days, the steady-state peripheral arterial blood flow and venous compliance decreased [39].

What's more, data from the $-6^{\circ}$ HDT experiment of the "Earth Star-1" project of China Astronaut Research and Training Center indicate that a sharp decrease of the lower venous compliance occurs, together with an increase of the total peripheral vascular resistance [40]. The differences between the conclusions lie in the determination of the venous compliance of the lower limbs. Traditionally, variation of the lower-limb venous compliance is determined by the change rate of the lower-limb volume. But the change rate of the lower-limb volume is affected by many factors such as the venous system, the muscle and body fluid, therefore the change rate of the lower-limb volume cannot reflect the changes of venous compliance correctly [41].

A result of the elevated venous compliance in weightlessness is that more blood will stay in the lower limbs of humans (standing posture), thus the blood flow returning to the right atrium decreases, which further results in the decreased cardiac output and mean arterial blood pressure. Therefore, the alterations of the compliance of the veins in lower extremities are the potential mechanism of the orthostatic intolerance after exposure to real and simulated weightlessness [42]. Evidences from the recent spaceflight 
have validated the idea that capacity of leg veins increases in weightlessness. Data from the MIR station indicates that the maximum capacity of leg veins increases during the 1st week, and the change becomes more pronounced after 30-40 days and is followed by a relative stabilization of hemodynamics at rest [30]. What's more, analysis of the hemodynamic data over 20 years from 26 astronauts on Salyut 7 and MIR station was conducted, and the results showed that the resistance of the lower-limb vessels decreased, but the capacity of the leg veins increased. And the LBNP test revealed the reduction of the gravitydependent reactions which got more conspicuous as flight duration extended further [43].

Multifactorial explanation for the changes in lower-limb veins has been put forward by researchers as below [44]. Changes of the hydrostatic pressure induced by weightlessness cause the decreased venous pressure in the lower body. Meanwhile, inactivity and the muscle atrophy in weightlessness result in the decreased blood flow in veins of the lower limbs. Moreover, decreased gravitational stimulations to the cardiovascular system in weightlessness lead to a weakened auto-regulation of the veins. The combined effects of these changes finally result in the decreased contractile response of the smooth muscle, reduced innervations and the atrophy of the vessel wall, which further bring about the alterations of the veins in lower extremities in weightlessness.

\section{Upper body vessel regulation}

In the researches concerning the upper body vascular system, more attention has been paid to the cerebral circulation. The auto-regulation of the cerebral vasculature guarantees the normal blood flow and blood pressure in the head, even in weightlessness. As reported in the previous parts of this literature review, blood shifts to the upper body in weightlessness, therefore the blood flow velocity, blood filling and vascular resistance in the head are supposed to be different from that on Earth. As for the changes of blood flow velocity, most work has been focused on the flow velocity in the middle cerebral artery. But there are discrepancies till now. Data from the Neurolab space shuttle mission suggested that the middle cerebral artery blood flow velocity of 6 astronauts at rest did not change significantly from the preflight values during or after spaceflight [45]. However, some other authors have come to opposite conclusions $[46,47]$. The differences may result from the experimental methods, posture, and physiological differences of the astronauts, therefore further investigations are still needed in the future.

In addition, the $-6^{\circ}$ HDT experiments showed that the systolic blood flow velocity and mean blood flow velocity in the right middle cerebral artery decreased significantly compared with the pre-HDT values, while the systolic and mean blood flow velocity in the left middle cerebral artery increased at the beginning of the experiments but decreased thereafter [48]. The finding is in good agreement with the conclusion presented by Satake in 1994 [49]. In addition to the blood flow velocity and blood filling, the blood pressure and cerebral resistance are also influenced by the weightlessness. The computational simulation conducted by Feng [50] in 2006 indicates that the cerebral blood flow elevates by $10 \%$ and the carotid artery pressure increases by $10 \mathrm{~mm} \mathrm{Hg}$ in weightlessness, compared with the values in the terrestrial circumstances [51]. During spaceflight, elevated cerebral artery blood pressure has been observed, with the blood congestion in the jugular veins [52]. And the cerebral venous congestion is also observed in simulated weightlessness. For example, $-6^{\circ} \mathrm{HDT}$ experiments recorded by Kawai and Marshall-Bowman suggest that the venous return decreases and more blood stays in the cerebral and jugular veins [53,54].

As for the changes in cerebral vascular resistance in weightlessness, there are different opinions. For example, Arbeille et al. observed reduced cerebral vascular resistance in 1 astronaut in the middle of a 25-day spaceflight, though they had also seen the tendency for 
increased carotid resistance index in 2 of 3 astronauts during a 237-day spaceflight on Salyut [55]. And Zhang reported a decreased femoral vascular resistance and an insignificant change in the cerebral vascular resistance during a 6-month spaceflight [56]. However, most findings in real and simulated weightlessness reveal that the cerebral vascular resistance increases. For example, increased cerebral vascular resistance and decreased cerebral blood flow were observed by Yang after a 21-day $-6^{\circ}$ HDT experiment [57]. And Marshall-Bowman [54] noted that the cerebral perfusion pressure decreased and cerebral vascular resistance elevated in long-term spaceflight, which resulted in reduced cerebral blood flow.

Except the cerebral circulation, studies on the upper body vessels also comprise the vascular regulations in upper extremities. As the fluid shifts from the lower to the upper body in weightlessness, there is an increase of fluid volume in the forearms, but the pulsed blood filling and vascular resistance are found unchanged. However, Nagatomo [58] reported recently that blood flow in the upper extremities did not change. In addition, studies by others have indicated a decrease in the vascular tone of the forearm during spaceflight [37].

\section{CONCLUSIONS AND POTENTIAL DIRECTIONS}

In conclusion, this review demonstrates that the weightlessness, either real or simulated, has significant effects on the cardiac and peripheral vascular functions, and induces a series of adaptive changes in the human body. These changes, such as altered cardiac contractile ability, altered cardiac rhythm and regulation of the vessels both in upper and lower body, result in risks to the cardiovascular system of the astronauts. With the extended flight duration, elevated activity intensity, and increased EVA, the health and safety of the astronauts are increasingly challenged during the spaceflight. Therefore, studies concerning the cardiovascular alterations induced by weightlessness are still necessary.
Moreover, potential directions that are closely related to the health, safety and working performance of the astronauts are put forward in this review. First, study and development of the countermeasures against the adverse effects of weightlessness, such as the reduced aerobic capacity and orthostatic intolerance, are still important in the future. Second, changes in the autonomic nerve induced by weightlessness have significant impacts on the heart and peripheral vessels of humans, and further research is still needed to determine the mechanism. Third, more attention should be paid to the mechanism and measures intended to prevent cardiac arrhythmia caused by weightlessness.

Finally, little research has been done on the thermal comfort of the astronauts during spaceflight. Actually, the thermal environment in the spacecraft and the thermal regulation (closely related to the cardiovascular system) of the humans in weightlessness greatly differ from that on Earth. Therefore, more attention should be paid in the future to studies on thermal comfort of the humans in weightlessness.

\section{ACKNOWLEDGMENTS}

The authors would like to express their appreciation to the National Natural Science foundation of China and Hunan Provincial Innovation Foundation for the financial support for this study.

\section{REFERENCES}

1. Iskovitz I, Kassemi M, Thomas JD. Impact of weightlessness on cardiac shape and left ventricular stress/strain distributions. J Biomech Eng. 2013;135(12):121008, http://dx.doi. org/10.1115/1.4025464.

2. Summers RL, Weston S, Ryan G, Jun L, Benjamin CW, Sourav P. Influence of microgravity on left ventricular sphericity: A finite element model of the heart. Proceedings of the 42nd International Conference on Environmental System; 2012 Jul 15-19; San Diego, USA. San Diego: American Institute of Aeronautics and Astronautics; 2012, p. 1-7, http://dx.doi.org/10.2514/6.2012-3448. 
3. Summers RL, Martin DS, Platts SH, Mercado-Young R, Coleman TG, Kassemi M et al. Ventricular chamber sphericity during spaceflight and parabolic flight intervals of less than 1 G. Aviat Space Environ Med. 2010;81(5):506-10, http://dx.doi.org/10.3357/ASEM.2526.2010.

4. Perhonen MA, Franco F, Lane LD, Buckey JC, Blomqvist CG, Zerwekh JE, et al. Cardiac atrophy after bed rest and spaceflight. J Appl Physiol. 2001;91(2):645-53.

5. Henry WL, Epstein SE, Griffith JM, Goldstein RE, Redwood DR. Effect of prolonged space flight on cardiac function and dimensions. Bethesda (US): National Institution of Health; Report No.: 19770026871. Contract No.: 77N33815. Sponsored by the NASA.

6. Summers RL, Martin DS, Meck JV, Coleman TG. Mechanism of spaceflight-induced changes in left ventricular mass. Am J Cardiol. 2005;95(9):1128-30, http://dx.doi. org/10.1016/j.amjcard.2005.01.033.

7. Hughson RL. Recent findings in cardiovascular physiology with space travel. Respir Physiol Neurobiol. 2009;169(Suppl 1):S38-41.

8. Summers RL, Martin DS, Meck JV, Coleman TG. Computer systems analysis of spaceflight induced changes in left ventricular mass. Comput Biol Med. 2007;37(3):358-63, http:// dx.doi.org/10.1016/j.compbiomed.2006.04.003.

9. Verheyden B, Liu J, Beckers F, Aubert AE. Adaptation of heart rate and blood pressure to short and long duration space missions. Respir Physiol Neurobiol. 2009;169(Suppl 1):S13-6.

10. Gundel A, Drescher J, Spatenko YA, Polyakov VV. Changes in basal heart rate in spaceflights up to 438 days. Aviat Space Environ Med. 2002;73(1):17-21.

11. Charles JB, Frey MA, Fritsch-Yelle JM, et al. Cardiovascular and cardiorespiratory function. In: Nicogossian AE, Mohler SR, Gazenko OG, Grigoriev AI, editors. Space biology and medicine. Reston (VA): American Institute of Aeronautics and Astronautics; 1996. p. 63-88.

12. Hamilton DR, Sargsyan AE, Martin DS, Garcia KM, Melton SL, Feiveson A, et al. On-orbit prospective echocardiography on International Space Station crew. Echocardiography. 2011;28(5):491-501, http://dx.doi.org/ 10.1111/j.1540-8175.2011.01385.x.

13. Hughson RL, Shoemaker JK, Blaber AP, Arbeille P, Greaves DK, Pereira-Junior PP, et al. Cardiovascular regulation during long-duration spaceflights to the International Space Station. J Appl Physiol. 2012;112(5):719-27, http:// dx.doi.org/10.1152/japplphysiol.01196.2011.

14. Carvil P, Baptista R, Russomano T. The human body in a microgravity environment: Long term adaptations and countermeasures. Aviat Foc. 2013;4(1):10-22.

15. Zuj KA, Arbeille P, Shoemaker JK, Blaber AP, Greaves DK, $\mathrm{Xu} \mathrm{D}$, et al. Impaired cerebrovascular auto-regulation and reduced $\mathrm{CO}_{2}$ reactivity after long-duration spaceflight. Am J Physiol Heart Circ Physiol. 2012;302(12):H2592-8.

16. Platts SH, Martin DS, Stenger MB, Perez SA, Ribeiro LC, Summers R, et al. Cardiovascular adaptations to longduration head-down bed rest. Aviat Space Environ Med. 2009;80(5 Suppl):A29-36.

17. Aubert AE, Verheyden B, d'Ydewalle C, Beckers F, van den Bergh O. Effects of mental stress on autonomic cardiac modulation during weightlessness. Am J Physiol Heart Circ Physiol. 2010;298(1):H202-9.

18. Romanov EM, Artamonva NP, Golubchikova ZA, Zavadovskii AF, Korotaev MM. [Results of long-term electrocardiographic examinations of cosmonauts]. Kosm Biol Aviakosm Med. 1987;21:10-4. Russian.

19. Rossum AC, Wood ML, Bishop SL, Deblock H, Charles JB. Evaluation of cardiac rhythm disturbances during extravehicular activity. Am J Cardiol. 1997;79(8):1153-5, http:// dx.doi.org/10.1016/S0002-9149(97)00071-4.

20. Baevsky RM, Baranov VM. Funtova II, Diedrich A, Pashenko AV, Chernikova AG, et al. Autonomic cardiovascular and respiratory control during prolonged spaceflights aboard the International Space Station. J Appl Physiol. 2007;103(1): 156-61, http://dx.doi.org/10.1152/japplphysiol.00137.2007.

21. Mitchell BM, Meck JV. Short-duration spaceflight does not prolong QTc intervals in male astronauts. 
Am J Cardiol. 2004;93(8):1051-2, http://dx.doi.org/10.1016/ j.amjcard.2003.12.060.

22. Anzai T, Frey MA, Nogami A. Cardiac arrhythmias during long-duration spaceflights. J Arrhythm. 2014;30(3):139-49, http://dx.doi.org/10.1016/j.joa.2013.07.009.

23. D'Aunno DS, Dougherty AH, DeBlock HF, Meck JV. Effect of short- and long-duration spaceflight on QTc intervals in healthy astronauts. Am J Cardiol. 2003;91(4):494-7, http:// dx.doi.org/10.1016/S0002-9149(02)03259-9.

24. Chinushi M, Furushima H, Hosaka Y, Komura S, Sato A, Iijima K, et al. Endocardial arrhythmogenic mechanisms of torsades de pointes in patients with the congenital long QTc syndrome. Intern Med. 2011;50(6):1695-702, http://dx.doi.org/10.2169/internalmedicine.50.5114.

25. Eckberg DL, Halliwill JR, Beightol LA, Brown TE, Taylor JA, Goble R. Human vagal baroreflex mechanisms in space. J Physiol. 2010;588(7):1129-38, http://dx.doi. org/10.1113/jphysiol.2009.186650.

26. William HC, Victor AC. Sympathetic nervous system and spaceflight. Acta Astronaut. 2007;60(4-7):223-33, http:// dx.doi.org/10.1016/j.actaastro.2006.08.003.

27. Akoum NW, Sanders NA, Wasmund SL, Hamdan MH. Irregular ventricular activation results in QT prolongation and increased QT dispersion: A new insight into the mechanism of AF-induced ventricular arrhythmogenesis. J Cardiovasc Electrophysiol. 2011;22(11):1249-52, http://dx.doi. org/10.1111/j.1540-8167.2011.02110.x.

28. Fritsch-Yelle JM, Leuenberger UA, D'Aunno DS, Rossum AC, Brown TE, Wood ML, et al. An episode of ventricular tachycardia during long-duration spaceflight. Am J Cardiol. 1998;81(11):1391-2, http://dx.doi.org/10.1016/ S0002-9149(98)00179-9.

29. Liu ZX, Li ZL, Wang DS, et al. [Effects of simulated microgravity on the gap junction protein expression spectrum in rat myocardium]. Space Med Med Eng. 2008;21(1):6-10. Chinese.

30. Fomina GA, Kotovskaia AR, Temnova EV. Dynamics of human cardiovascular responses in different periods of long-term exposure to weightlessness. Hum Physiol. 2012;38(7):715-20, http://dx.doi.org/10.1134/S0362119712 070080 .

31. Arbeille P, Fomina G, Roumy J, Alferova I, Tobal N, Herault S. Adaptation of the left heart, cerebral and femoral arteries, and jugular and femoral veins during short- and longterm head-down tilt and spaceflights. Eur J Appl Physiol. 2001;86(2):157-68, http://dx.doi.org/10.1007/s004210100473.

32. Fomina GA, Kotovskaya AR, Pochuev VI, Zhernavkov AF. Mechanisms of changes in human hemodynamics under the conditions of microgravity and prognosis of postflight orthostatic stability. Hum Physiol. 2008;34(3):92-7, http:// dx.doi.org/10.1134/S0362119708030122.

33. Hughson RL, Shoemaker JK, Arbeille P. CCISS, vascular and BP reg: Canadian space life science research on ISS. Acta Astronaut. 2014;104(1):444-8.

34. Tuday EC, Nyhan D, Shoukas AA, Berkowitz DE. Simulated microgravity-induced aortic remodeling. J Appl Physiol. 2009;106(6):2002-8, http://dx.doi.org/10.1152/japplphy siol.90777.2008.

35. Tuday EC. Effects of microgravity exposure on arterial vascular stiffness [dissertation]. Maryland: The Johns Hopkins University; 2009.

36. Watenpaugh DE, Buckey JC, Lane LD, Gaffney FA, Levine $\mathrm{BD}$, Moore WE, et al. Effects of spaceflight on human calf hemodynamics. J Appl Physiol. 2001;90(4):1552-8.

37. Watenpaugh DE, Hargens AR. The cardiovascular system in microgravity. In: Fregly MJ, Blatteis CM, editors. Handbook of physiology: Environmental physiology. New York: Oxford University Press; 1996. p. 640-2, http://dx.doi.org/10.1002/ cphy.cp040129.

38. Fomina GA, Kotovskaia AR. [Shifts in human venous hemodynamics in long-term space flight]. Aviakosm Ekolog Med. 2005;39(4):25-30. Russian.

39. Christ F, Gamble J, Baranov V, Kotov A, Chouker A, Thiel M, et al. Changes in microvascular fluid filtration capacity during 120 days of 6 degrees head-down tilt. J Appl Physiol. 2001;91(6):2517-22. 
40. Ying-Hui L, Yu-Min W, Yan-Qiang B, Shi-Zhong J, Yi-Bing D, Shan-Guang C. [Outline of "Earth Star-1" 60-d head-down bed rest experiment]. Space Med Med Eng. 2008;21(3):291-4. Chinese.

41. Yue Y. Changes in compliance of femoral vein after simulated weightlessness in rabbits with histomorphology correlation [dissertation]. Xi'an: The Fourth Military Medical University; 2003.

42. Xi-Qing S. Microgravity-induced cardiovascular deconditioning: Mechanisms and countermeasures. Zhongguo Ying Yong Sheng Li Xue Za Zhi. 2012;28(6):532-9.

43. Kotovskaia AR, Fomina GA. [The features of adaptation and disadaptation of the human cardiovascular system in the space flight conditions]. Fiziol Cheloveka. 2010;36:78-86. Russian.

44. Liang WB, Jiang SZ, Shen XY. [Changes of leg compliance during weightlessness or simulated weightlessness]. Space Med Med Eng. 2002;15(2):140-3. Chinese.

45. Iwasaki K, Levine BD, Zhang R, Zuckerman JH, Pawelczyk JA, Diedrich A, et al. Human cerebral autoregulation before, during and after spaceflight. J Physiol. 2007;579(3): 799-810, http://dx.doi.org/10.1113/jphysiol.2006.119636.

46. Arbeille P, Kerbeci P, Greaves D, , Schneider S, Hargens A, Hughson R. Arterial and venous response to tilt with LBNP test after a 60 day HDT bed rest. J Gravit Physiol. 2007;14(1):47-8.

47. Sun XQ, Yao YJ, Yang CB, Jiang SZ, Jiang CL, Liang WB. Effect of lower-body negative pressure on cerebral blood flow velocity during 21 days head-down tilt bed rest. Med Sci Monit. 2005;11(1):1-5.

48. Wang ZB. [Hemodynamic measure on human cerebral artery by transcranial Doppler at simulated weightlessness]. Chin J Convalescent Med. 2009;18(5):391-4. Chinese.

49. Satake H, Konishi T, Kawashima T, Matsunami K, Uno T, Imai S, et al. Intracranial blood flow measured with single photon emission computer tomography (SPECT) during transient -6 degrees head-down tilt. Aviat Space Environ Med. 1994;65(2):117-22.

50. Feng DY, Sun XQ, Lu HB. [Simulation study on cerebral blood flow during weightlessness]. Space Med Med Eng. 2006;19(3):163-6. Chinese.

51. Wu DW, Shen XY. [Changes of cerebral circulation during weightlessness or simulated weightlessness]. Space Med Med Eng. 2000;13(5):386-90. Chinese.

52. Khurana I. Textbook of medical physiology. Noida: Elsevier; 2006.

53. Kawai Y, Doi M, Setogawa A, Shimoyama R, Ueda K, Asai Y, et al. Effects of microgravity on cerebral hemodynamics. Yonago Acta Med. 2003;46:1-8.

54. Marshall-Bowman K. Increased intracranial pressure and visual impairment associated with long duration space-flight [dissertation]. Illkirch-Graffenstaden: International Space University; 2011.

55. Arbeille P, Shoemaker K, Kerbeci P, Schneider S, Hargens A, Hughson R. Aortic, cerebral and lower limb arterial and venous response to orthostatic stress after a 60-day bed rest. Eur J Appl Physiol. 2012;112(1):277-84, http://dx. doi.org/10.1007/s00421-011-1935-y.

56. Zhang F-L. Vascular adaptation to microgravity: What have we learned? J Appl Physiol. 2001;91(6):2415-30.

57. Yang CB, Yao YJ, Sun XQ. Effect of lower body negative pressure on cardiac function and cerebral blood flow during $21 \mathrm{~d}$ head-down tilt bed rest. J Fourth Mil Med Univ. 2001;22(20):1903-8.

58. Nagatomo F, Kouzaki M, Ishihara A. Effects of microgravity on blood flow in the upper and lower limbs. Aerosp Sci Technol. 2014;34:20-3, http://dx.doi.org/10.1016/j.ast. 2014.01.009.

This work is available in Open Access model and licensed under a Creative Commons Attribution-NonCommercial 3.0 Poland License - http://creativecommons.org/ licenses/by-nc/3.0/pl/deed.en. 